\title{
The Problem of Recurrence for Lorentz Processes
}

\author{
András Krámli ${ }^{1}$ and Domokos Szász ${ }^{2}$ \\ 1 Computer and Automation Institute, Hungarian Academy of Sciences, Budapest, Hungary \\ 2 Mathematical Institute, Hungarian Academy of Sciences, Budapest, Hungary
}

Dedicated to Professor Ya. G. Sinai on the occasion of his 50th birthday

\begin{abstract}
We prove that, for the planar Lorentz process with a periodic configuration of scatterers, the quasi-local CLT of the gaussian $\left\{\log ^{e} n\right\}$ type holds for any $\varrho>1$. Consequently, for arbitrary $\varrho>3 / 2$, the probabilities that, at the moment of the $n$th reflection, this process lies in a square of size $\log ^{e} n$ are asymptotically gaussian. This implies that these events occur for infinitely many values of $n$ (i.e. a weaker form of recurrence).
\end{abstract}

\section{Introduction and Main Results}

There is a general belief that the statistical properties of the Lorentz process are quite similar to those of a random walk. The theoretical support of this belief becomes interesting from a physical point of view by the fact that the Lorentz process is an intermediate model between the mechanical Brownian motion and the random walks of probability theory. Moreover, recent sophisticated techniques like Markov partitions not only make certain important properties of the Lorentz process rigorously tractable but they are also hoped to become the right methods for less simplified models.

At present, however, the Markov partition of the Sinai billiard [B-S(1980)] only enables us to treat the probabilistic behaviour of the planar Lorentz process with a periodic configuration of scatterers.

In fact, first the central limit theorem was proved by Bunimovich and Sinai [B-S(1981)] who built a Markov approximation for the Lorentz process and composed it with Bernstein's classical method. Later the present authors approximated the Lorentz process with random walks with internal states and their method also gives Cramér-Edgeworth-Tchebysheff type expansions in the central limit theorem $[\mathrm{K}-\mathrm{Sz}(1983)] \mathrm{b})$.

In 1979 Sinai proposed to study the recurrence properties of the Lorentz process which are, in general, strongly connected with local versions of central limit theorems. He conjectured that the Lorentz process also obeys the statement of Pólya's classical theorem: it is recurrent on the plane and it is transient in higher 
dimensions. The lacunary feature of the Doeblin property of the Markov partition (cf. Sect. 2), has however, hindered us in obtaining a local central limit theorem for the Lorentz process which, in general, is the usual tool when studying recurrence properties.

Here we introduce and prove quasi-local central limit theorems for Lorentz processes.

Definition 1.2. We say that a sequence $\left\{S_{n}\right\}$ of random vectors satisfies a quasi-local central limit theorem of the gaussian $\left\{a_{n}\right\}$ type if

(i) there exists a sequence $\left\{\eta_{n}\right\}$ of random vectors such that $\eta_{n}$ has a gaussian distribution with mean 0 and covariance matrix $a_{n}^{2} I$ ( $I$ is the unit matrix);

(ii) $S_{n}+\eta_{n}$ obeys the local central limit theorem.

This definition makes sense if $\left\{a_{n}\right\}$ is an increasing sequence of positive numbers such that $a_{n} \rightarrow \infty$ and $a_{n}=o(\sqrt{n})$. The case $a_{n}=$ const would mean a local limit theorem while the choice $a_{n}=$ const $\sqrt{n}$ would already change the form of the global central limit theorem.

The main result of this paper is Theorem 3.1. To formulate it we consider the symbolic representation $\left(\Omega, \tilde{\psi}, \mu_{0} T_{0}\right)$ of the Sinai billiard, and we take a nice bounded function $h: \Omega \rightarrow \mathbb{R}$. Then under some additional assumption we have

Theorem 3.1. The partial sums $S_{n}=\sum_{k=0}^{n-1} h\left(T_{0} \omega\right)$ obey the quasi-local central limit theorem of the gaussian $\left\{\log ^{\varrho} n\right\}$ type for any $\varrho>1(d=2)$.

The proof of this theorem combines the analytic method of characteristic functions with direct probabilistic ideas.

If the symbolic dynamics will be constructed for higher dimensional Sinai billiards, then our method will extend the validity of the QLCLT for higher dimensions, too, and thus we can immediately obtain the transience of the Lorentz process for $d \geqq 3$. Therefore the paper is formulated in a general $d$-dimensional setup.

Since our quasi-local central limit theorems do not imply the local ones, we can only prove quasi-recurrence in dimension 2 .

Definition 4.1. We say that a sequence of random vectors $S_{n}$ is quasi-recurrent of the $\left\{a_{n}\right\}$ type if

where

$$
P\left(S_{n} \in Q\left(a_{n}\right) \text { i.o. }\right)=1,
$$

$$
Q(r)=\left\{\left(y_{1}, \ldots, y_{d}\right):\left|y_{1}\right|, \ldots,\left|y_{d}\right| \leqq r\right\} .
$$

Theorem 4.2. The two-dimensional Lorentz process is quasi-recurrent of the $\left\{\log ^{e} n\right\}$ type for any $\varrho>\frac{3}{2}$.

The presentation of the results is the following. The notions and some useful properties of quasi-local central limit theorems are treated in Sect. 1. Section 2 contains the necessary information on the Markov partition of the Sinai billiard. In Sect. 3 we formulate and prove our quasi-local central limit theorem for the Lorentz process and in Sect. 4 we describe the recurrence properties of Lorentz processes. 


\section{Quasi-Local Central Limit Theorems}

The difference between global and local versions of central limit theorems (briefly CLT and LCLT) is that they refer to asymptotic probabilities of sets increasing like $\sqrt{n}$ ( $n$ is the number of summands) and of constant sets respectively. We introduce quasi-local central limit theorems (QLCLT) to describe an intermediate case for sequences of sets increasing not slower than a suitable rate. In the sequel we will consider random vectors in $\mathbb{R}^{d}$ and $\sigma$ will denote a $d$-dimensional covariance matrix.

Definition 1.1. We say that a sequence $\left\{S_{n}\right\}$ of random vectors satisfies the QLCLT $(\sigma)$ of the bounded $\left\{a_{n}\right\}$ type if $\left\{a_{n}\right\}$ is an increasing sequence of positive numbers and there exists a sequence $\left\{\eta_{n}\right\}$ of random vectors such that

$$
P\left(\left|\eta_{n}\right| \leqq a_{n}\right)=1,
$$

and $S_{n}+\eta_{n}$ obeys the LCLT $(\sigma)$.

In what follows we always suppose $a_{n}=o\left(n^{1 / 2}\right)$, since otherwise the notions loose their local character.

We recall that a sequence of absolutely continuous random vectors with densities $p_{n}(x),\left(x \in \mathbb{R}^{d}\right)$ obeys the $\operatorname{LCLT}(\sigma)$ if

$$
\lim _{n \rightarrow \infty} \sup _{x}\left|n^{d / 2} p_{n}\left(n^{1 / 2} x\right)-\varphi_{\sigma}(x)\right|=0,
$$

where $\varphi_{\sigma}(x)$ is the gaussian density with mean 0 and covariance matrix $\sigma$. A simple consequence of this property is that then

$$
\lim _{n \rightarrow \infty} \int_{\mathbb{R}^{d}}\left|n^{d / 2} p_{n}\left(n^{1 / 2} x\right)-\varphi_{\sigma}(x)\right| d x=0
$$

(cf. [I-L (1965)]).

Definition 1.2. We say that a sequence $\left\{S_{n}\right\}$ of random vectors satisfies the QLCLT $(\sigma)$ of the gaussian $\left\{a_{n}\right\}$ type if there exists a sequence $\left\{\eta_{n}\right\}$ of random vectors such that $\eta_{n}$ has a gaussian distribution with mean 0 and covariance matrix $a_{n}^{2} I$ ( $I$ is the unit matrix) and, moreover, $S_{n}+\eta_{n}$ obeys the LCLT.

The heart of these definitions is reflected by the following simple propositions. Since we are considering quasi-local central limit theorems with a definite application in mind, we do not want to elaborate their detailed theory here.

Proposition 1.3. If the sequence $\left\{S_{n}\right\}$ of random vectors obeys the QLCLT $(\sigma)$ of the bounded $\left\{a_{n}\right\}$ type, then for every sequence $0 \leqq \ell_{n} \leqq \ell_{n+1}, \lim _{n \rightarrow \infty} \ell_{n}=\infty$ and arbitrary $x_{n} \in \mathbb{R}^{d}$,

$$
P\left(S_{n} \in x_{n}+Q\left(\ell_{n} a_{n}\right)\right) \sim \phi_{\sigma}\left[n^{-1 / 2}\left(x_{n}+Q\left(\ell_{n} a_{n}\right)\right)\right],
$$

where $\phi_{\sigma}$ denotes the gaussian distribution with density $\varphi_{\sigma}$.

Proof. Since

$$
P\left(S_{n} \in x_{n}+Q\left(\ell_{n} a_{n}\right)\right)=P\left(S_{n}+\eta_{n} \in x_{n}+Q\left(\ell_{n} a_{n}\right)+\eta_{n}\right),
$$


by Definition 1.1 we have

$$
\begin{aligned}
P\left(S_{n}+\eta_{n} \in x_{n}+Q\left[\left(\ell_{n}-1\right) a_{n}\right]\right) & \leqq P\left(S_{n} \in x_{n}+Q\left(\ell_{n} a_{n}\right)\right) \\
& \leqq P\left(S_{n}+\eta_{n} \in x_{n}+Q\left[\left(\ell_{n}+1\right) a_{n}\right]\right) .
\end{aligned}
$$

But $S_{n}+\eta_{n}$ obeys the $\operatorname{LCLT}(\sigma)$, and consequently its density tends to $\varphi_{\sigma}$ is variation. Hence the statement.

Proposition 1.4. If the sequence $\left\{S_{n}\right\}$ of random vectors satisfies the QLCLT $(\sigma)$ of the gaussian $\left\{a_{n}\right\}$ type and $0 \leqq \ell_{n} \leqq \ell_{n+1}$ with $(\log n)^{-1 / 2} \ell_{n} \rightarrow \infty$, then

$$
P\left(S_{n} \in Q\left(\ell_{n} a_{n}\right) \sim \phi_{\sigma}\left[n^{-1 / 2} Q\left(\ell_{n} a_{n}\right)\right] .\right.
$$

Proof. We will use the obvious bound

$$
P\left(\left|\eta_{n}\right| \geqq a_{n} K\right) \leqq e^{-c K^{2}},
$$

where $c>0$. Thus

$$
\begin{aligned}
P\left(S_{n} \in Q\left(\ell_{n} a_{n}\right)\right) & =P\left(S_{n}+\eta_{n} \leqq Q\left(\ell_{n} a_{n}\right)+\eta_{n}\right) \\
& =P\left(S_{n}+\eta_{n} \in Q\left(\ell_{n} a_{n}\right)+\eta_{n} ;\left|\eta_{n}\right| \leqq a_{n} K_{n}\right)+\vartheta_{n},
\end{aligned}
$$

where $\vartheta_{n} \leqq \exp \left(-c K_{n}^{2}\right)$.

Now similarly as in the previous proof we obtain

$$
\begin{aligned}
& P\left(S_{n}+\eta_{n} \in Q\left[\left(\ell_{n}-K_{n}\right) a_{n}\right]\right)-2 \vartheta_{n} \\
& \quad \leqq P\left(S_{n} \in Q\left(\ell_{n} a_{n}\right)\right) \leqq P\left(S_{n}+\eta_{n} \in Q\left[\left(\ell_{n}+K_{n}\right) a_{n}\right]\right)+2 \vartheta_{n} .
\end{aligned}
$$

This chain gives the statement whenever $K_{n}=o\left(\ell_{n}\right)$ and

$$
\vartheta_{n}=o\left(P\left(S_{n} \in Q\left(\ell_{n} a_{n}\right)\right)\right)
$$

hold. These orders can, however, be easily reached since $(\log n)^{-1 / 2} \ell_{n} \rightarrow \infty$.

The next proposition shows the usefulness of Definition 1.2: even without controlling the behavior of the far tails of the characteristic functions we can have a QLCLT.

\section{Proposition 1.5. If}

(i) $(\log n)^{-1 / 2} a_{n} \rightarrow \infty$,

(ii) $n^{-1 / 2} S_{n}$ satisfies the $\operatorname{CLT}(\sigma)$,

(iii) there exist positive constants $A, \varepsilon, c$ such that for every $n$ and $A \leqq\|t\| \leqq \varepsilon n^{1 / 2}$, $\left(t \in \mathbb{R}^{d}\right)$,

$$
\mid E \exp \left(\text { in }^{-1 / 2}\left(t, S_{n}\right)\right) \mid \leqq g(t)
$$

where $\int_{A}^{\infty} g(t) d t<\infty$, then $S_{n}$ obeys the QLCLT $(\sigma)$ of the gaussian $\left\{a_{n}\right\}$ type.

Proof. We show that, in Definition 1.2, the gaussian variables $\eta_{n}$ can be chosen independent of $S_{n}$. Then, by classical knowledge, it is sufficient to prove that

$$
\lim _{n \rightarrow \infty} \int\left|f_{n}(t) \exp \left(-\frac{a_{n}^{2}}{2 n}\|t\|^{2}\right)-\varphi_{\sigma}(t)\right| d t=0,
$$


where $f_{n}(t)=E \exp \left[\operatorname{in}^{-1 / 2}\left(t, S_{n}\right)\right]$ (cf. [I-L (1965)]). The expression on the left-hand side can be bounded in the usual way by the sum

$$
\begin{aligned}
& \int_{\|t\|<A}\left|f_{n}(t) \exp \left(-\frac{a_{n}^{2}}{2 n}\|t\|^{2}\right)-\varphi_{\sigma}(t)\right| d t \\
& \quad+\int_{\|t\|>A}\left|\varphi_{\sigma}(t)\right| d t+\int_{A \leqq t \leqq \varepsilon \sqrt{n}} \mid f_{n}(t) \exp \left(-\frac{a_{n}^{2}}{2 n}\|t\|^{2}\right) d t \\
& \quad+\int_{\|t\|>\varepsilon \sqrt{n}}\left|f_{n}(t)\right| \exp \left(-\frac{a_{n}^{2}}{2 n}\|t\|^{2}\right) d t=I_{1}+I_{2}+I_{3}+I_{4} .
\end{aligned}
$$

By (i) and (ii) $I_{1} \rightarrow 0$ if $n \rightarrow \infty$ for fixed $A$. On the other hand $I_{2} \rightarrow 0$ if $A \rightarrow \infty$. In view of condition (iii)

$$
I_{3} \leqq \int_{A<\|t\|<\varepsilon \sqrt{n}}\left|f_{n}(t)\right| d t \leqq \int_{\|t\|>A} g(t) d t .
$$

Finally

$$
\begin{aligned}
I_{4} & \leqq \int_{\|t\|>\varepsilon \sqrt{n}} \exp \left(-\frac{a_{n}^{2}}{2 n}\|t\|^{2}\right) d t \leqq \frac{n^{d / 2}}{a_{n}^{d}} \int_{\varepsilon a_{n}}^{\infty} e^{-\frac{u^{2}}{4}} d u \\
& \leqq \frac{n^{d / 2}}{a_{n}^{d}} e^{-\frac{\varepsilon^{2}}{4} a_{n}^{2}}
\end{aligned}
$$

and by (i) the right-hand side tends to 0 .

\section{Prerequisites: Properties of the Markov Partition of the Sinai-Billiard}

First we recall the necessary information on the Lorentz process and the Sinai billiard. The main notations will be adapted to [B-S (1981)].

Consider in $\mathbb{R}^{d}$ a $\mathbb{Z}^{d}$-periodic set of disjoint, smooth, strictly convex, closed scatterers. We suppose that there is no infinite line disjoint from the set of scatterers (i.e. the Lorentz process or the corresponding Sinai billiard has finite horizon). We label the congruent finite sets $(\mathscr{S})$ of the scatterers by $x \in \mathbb{Z}^{d}: \mathscr{S}_{x}$. The Lorentz process is the uniform motion of a point particle with elastic collisions at the scatterers. In other words, by considering it with the Liouville measure as an invariant measure it is a billiard system with a non-compact phase space (for a formal definition see [C-F-S (1982), Chap. 6]).

It is convenient to discretize the time and to study the Poincare map of the original, continuous time dynamical system. This map $T_{0}$ is defined on the 2(d-1)dimensional phase space $\mu_{1}=\bigcup_{x \in \mathbb{Z}^{d}} \tilde{\mathscr{S}}_{x}=\bigcup_{x \in \mathbb{Z}^{d}}$ boundary of $\mathscr{S}_{x} \otimes$ angle (in moments of relections). In a point $q$ of the boundary of $\mathscr{S}$ the angle $v$ can be identified with an element of $S^{d-1}(d-1$-dimensional unit surface) which satisfies $(v, n(q)) \geqq 0$, where $n(q)$ is the outer normal to $\mathscr{S}$ in $q \in \partial \mathscr{S} . T_{0}$ has a $\mathbb{Z}^{d}$-periodic invariant measure $\mu_{L}$ of the form $\delta \otimes \tilde{\mu}$, where $\delta$ is the uniform measure on $\mathbb{Z}^{d}$ and

$$
d \tilde{\mu}((q, v))=(v, n(q)) d \ell(q) d \omega(q),
$$

where $d \ell(q)$ is the Riemannian volume on $\partial \mathscr{S}$ and $d \omega(q)$ is the Lebesgue measure on $S^{d-1}$. 
By taking the Lorentz process $\bmod \mathbb{Z}^{d}$ we obtain a Sinai billiard on the $d$-dimensional torus. In [B-S (1980)] a Markov partition is constructed for the Poincaré map of the planar Sinai billiard and we will use its properties. Let us denote this discrete dynamical system by $\left(M_{1}, \tilde{\psi}, \mu_{0}, T_{0}\right)$, where $M_{1}, \mu_{0}$, and $T_{0}$ are just $M_{1}, \mu_{L}$, and $T_{0}$ taken $\bmod \mathbb{Z}^{d}$ and $\mu_{0}$ is also normed $\left(\mu_{0}\left(M_{1}\right)=1\right), \tilde{\psi}$ is the natural $\sigma$-algebra.

The Markov partition $\eta$ is a countable partition of

$$
M_{1}: M_{1}=\bigcup_{w \in W} A_{w},
$$

where $W$ is a countable index set. Let $\Omega$ be the space of sequences $\omega=\left\{\omega_{i}\right\}_{-\infty}^{\infty}$ where $\omega_{i} \in W$. We consider the map $\varphi: M_{1} \rightarrow \Omega$, where $\varphi(x)=\omega$ if $T_{0}^{n} x \in A_{\omega_{n}}$, $-\infty<n<\infty$. Two rank functions $r_{+}, r_{-}: W \rightarrow \mathbb{Z}_{+}$are also introduced. We shall briefly write $r_{+}(w)$ and $r_{-}(w)$ instead of $r_{+}\left(A_{w}\right)$ and $r_{-}\left(A_{w}\right)$. The measure $\varphi^{*} \mu_{0}$ is defined on the natural $\sigma$-algebra of subsets of $\Omega$, and it is shift invariant. We will denote it by $\mu_{0}$, too. Next we recall from [B-S (1981)] the Tail Bound, the Markov Approximation and the Doeblin Properties of the Markov partition, which are there proved for $d=2$.

Property $T B$. There exists $\lambda_{1} \in(0,1)$ such that for all sufficiently large $k$,

$$
\mu_{0}\left(\omega: r_{+}\left(\omega_{0}\right) \geqq k\right)+\mu_{0}\left(\omega: r_{-}\left(\omega_{0}\right) \geqq k\right) \leqq \lambda_{1}^{k} .
$$

For any constant $\lambda_{20}, \lambda_{21}, \lambda_{22} \in(0,1), \lambda_{20}<\lambda_{22}$, we introduce the subsets of $M_{1}$,

$$
\begin{aligned}
U_{m} & =\left\{x: \operatorname{dist}\left(x, \partial M_{1}\right)<\lambda_{20}^{m}\right\}, \quad m \in \mathbb{Z}_{+}, \\
V_{n} & =\left\{x: T_{0}^{k} x \notin U_{m}, m=\left[n^{\lambda_{21}}\right],|k| \leqq n ; T_{0}^{j} x \notin U_{j},|j|>n\right\}, \\
Z_{n} & =\left\{x: \mu_{0}\left(V_{n} \mid C_{\eta^{-}}(x)\right)>1-\lambda_{22}^{\sqrt{n}}\right\} .
\end{aligned}
$$

Here $\eta^{-}$is the partition of $\Omega$ arising if we fix all $\omega_{i},-\infty<i \leqq 0$, and $C_{\eta^{-}}(x)$ the element of $\eta^{-}$containing $\varphi(x)$. It is easy to see that

$$
\begin{gathered}
\mu_{0}\left(U_{m}\right)<\lambda_{20}^{m}, \quad \mu_{0}\left(\bar{V}_{n}\right) \leqq 2 n \lambda_{20}^{\left[n^{\left.\lambda_{21}\right]}\right.}+\lambda_{20}^{n} /\left(1-\lambda_{20}\right), \\
\mu_{0}\left(\bar{Z}_{n}\right)<\frac{2 n}{1-\lambda_{20}}\left(\frac{\lambda_{20}}{\lambda_{22}}\right)^{\left[n^{\left.\lambda_{21}\right]}\right.} .
\end{gathered}
$$

Property MA. Suppose that $C_{\eta^{-}}^{\tau}$ corresponds to $\omega_{0}, \omega_{-1}, \ldots, \omega_{-n+1}, \omega_{-n}^{\tau}$, $\omega_{-n-1}^{\tau}, \ldots ; \tau=1,2$, and $C_{\eta^{-}}^{\tau} \in \varphi\left(Z_{n}\right)$. Then one can choose $\lambda_{20}, \lambda_{21}, \lambda_{22}$, and $\lambda_{2} \in(0,1)$ in such a way that

$$
\sum_{\omega_{1}}\left|\mu_{0}\left(\omega_{1} \mid C_{\eta^{-}}^{1}\right)-\mu_{0}\left(\omega_{1} \mid C_{\eta^{-}}^{2}\right)\right| \leqq \lambda_{2}^{n^{1 / 2}} .
$$

Consider the conditional distributions

$$
\pi^{\tau}\left(\omega_{3 n+1}, \ldots, \omega_{4 n}\right)=\mu_{0}\left(\omega_{3 n+1}, \ldots, \omega_{4 n} \mid \omega_{0}^{\tau}, \ldots, \omega_{-n+1}^{\tau}\right),
$$

$\tau=1,2$ on the space of words $\omega_{3 n+1}, \ldots, \omega_{4 n}$.

Property $D$. Suppose that $r_{ \pm}\left(\omega_{i}^{\tau}\right) \leqq n, 1 \leqq i \leqq n, \tau=1,2$. Then there exists a constant $\lambda_{3} \in(0,1)$ such that for all $n$ large enough

$$
\operatorname{Var}\left(\pi^{1}, \pi^{2}\right)=\frac{1}{2} \sum_{\omega_{3 n+1}, ., \omega_{4 n}} \mid \pi^{1}\left(\omega_{3 n+1}, \ldots, \omega_{4 n}\right)-\pi^{2}\left(\omega_{3 n+1}, \ldots, \omega_{4 n} \mid \leqq \lambda_{3} .\right.
$$




\section{Quasi-Local Central Limit Theorem for the Lorentz-Process}

Suppose that $h$ is a bounded function on $\Omega\left(\|h\|_{\text {sup }}=C_{1}\right)$, and there exists a constant $\lambda_{4} \in(0,1)$ such that, for every sufficiently large $n$, one can find cylindric functions $h_{n}(\omega)=h_{n}\left(\omega_{-n}, \ldots, \omega_{n}\right), \int h_{n} d \mu_{0}=0$ depending only on the coordinates $\omega_{i},|i| \leqq n$ and satisfying

$$
\left\|h(\omega)-h_{n}(\omega)\right\|_{\text {sup }} \leqq \lambda_{4}^{n} .
$$

In [B-S (1981)] the following CLT was proved: if $d=2$ and

$$
\sigma_{h}=\sum_{n=-\infty}^{\infty} E\left(T_{0}^{n} h \cdot h\right)>0,
$$

then for every $a, b, a<b$,

$$
\lim _{n \rightarrow \infty} \mu_{0}\left(\omega: a<\left(\sigma_{h} n\right)^{-1 / 2} \sum_{k=0}^{n-1} h\left(T_{0}^{k} \omega\right)<b\right)=(2 \pi)^{-1} \int_{a}^{b} \exp \left(-\frac{u^{2}}{2}\right) d u .
$$

Under the same assumptions we have

Theorem 3.1. If $\sigma_{h}$ then the partial sums $\sum_{k=0}^{n-1} h\left(T_{0}^{k} \omega\right)$ obey the $\mathrm{QLCLT}$ of the gaussian $\left\{\log ^{1+\delta} n\right\}$ type for arbitrary $\delta>0$.

Applying this general theorem one easily obtains a corollary giving interesting inference on the Lorentz process. For $x_{0} \in M_{1}$ put $x_{1}=T x_{0}$ and $h^{(j)}\left(x_{0}\right)$ $=q^{(j)}\left(x_{1}\right)-q^{(j)}\left(x_{0}\right), 1 \leqq j \leqq d$. It is easy to see that $E h^{(j)}=0$ and $h^{(j)}$ satisfies the conditions formulated at the beginning of this section $(1 \leqq j \leqq d)$. Moreover, in [B-S (1981)] it is also shown that the covariance matrix $\sigma_{h}$ of the problem is nondegenerate.

Corollary 3.2. If $d \geqq 2$ and $\mathbf{h}=\left(h^{(1)}, \ldots, h^{(d)}\right)$ are as defined above, then the partial sums $\sum_{k=0}^{n-1} \mathbf{h}\left(T_{0}^{k} \omega\right)$ obey the QLCLT $\left(\sigma_{h}\right)$ of the gaussian $\left\{a_{n}=\log ^{1+\delta} n\right\}$ type for arbitrary $\delta>0$. In particular, for any sequence $\left\{\ell_{n}\right\}, 0 \leqq \ell_{n} \leqq \ell_{n+1}$ with $(\log )^{-1 / 2} \ell_{n} \rightarrow \infty$,

$$
\mu_{0}\left(n^{-1 / 2} \sum_{k=0}^{n-1} \mathbf{h}\left(T_{0}^{k} \omega\right) \in Q\left(\ell_{n} a_{n}\right)\right) \sim \phi_{\sigma_{h}}\left[n^{-1 / 2} Q\left(\ell_{n} a_{n}\right)\right] .
$$

Proof of Theorem 3.1. Our starting point is the proof of Lemma 5 in [B-S (1981)] and we use the notations of that paper. We consider the characteristic function

$$
\psi_{n}(t)=E \exp \left[i\left(\sigma_{h} n\right)^{-1 / 2} t \sum_{k=0}^{n-1} h\left(T_{0}^{k} \omega\right)\right] .
$$

We decompose the interval $[0, n-1]$ into disjoint subintervals

$$
\Delta=\Delta_{1}^{(1)} \cup \Delta_{1}^{(2)} \cup \Delta_{2}^{(1)} \cup \Delta_{2}^{(2)} \cup \ldots \cup \Delta_{p-1}^{(2)} \cup \Delta_{p}^{(1)} \cup \Delta_{p}^{(2)}
$$

in such a way that the length $\left|\Delta_{i}^{(1)}\right|$ of each $\Delta_{i}^{(1)}$ except the last one is equal to $A_{n}$ while the length of each $\Delta_{i}^{(2)}$ except the last one is equal to $B_{n}=o\left(A_{n}\right)$. To use 
Bernstein's classical method we will focus on

$$
\psi_{n}^{(1)}(t)=E \exp \left[i\left(\sigma_{h} n\right)^{-1 / 2} t \sum_{s=1}^{p} \sum_{k \in \Delta\left(\frac{1}{s}\right)} h\left(T_{0}^{k} \omega\right)\right]
$$

Thus we have omitted a term with

$$
\left|\sum_{s=1}^{p} \sum_{k \in \Delta(s)} h\left(T_{0}^{k} \omega\right)\right| \leqq \text { const } \cdot p \cdot B_{n} .
$$

Next we take a cylindric function $h_{n_{1}}$ according to the introductory words of this section, and thus we have $\int h_{n_{1}} d \mu_{0}=0$ and

$$
\left|\sum_{s=1}^{p} \sum_{k \in \Delta_{s}^{(1)}}\left(h\left(T_{0}^{k} \omega\right)-h_{n_{1}}\left(T_{0}^{k} \omega\right)\right)\right| \leqq \operatorname{const} n \lambda_{4}^{n_{1}} .
$$

We will consider

$$
\psi_{n}^{(2)}(t)=E \exp \left[i\left(\sigma_{h} n\right)^{-1 / 2} t \sum_{s=1}^{p} \sum_{k \in \Delta\left(\frac{1}{s}\right)} h_{n_{1}}\left(T_{0}^{k} \omega\right)\right] .
$$

Lemma 3.3. The sums $S_{n}=\sum_{s=1}^{p} \sum_{k \in \Delta(1)} h_{n_{1}}\left(T_{0}^{k} \omega\right)$ satisfy conditions (ii) and (iii) of Proposition 1.5 and if $(\log n)^{-1 / 2} a_{n} \rightarrow \infty$ but $a_{n}^{2}=o(n)$, then $S_{n}$ obeys the QLCLT $\left(\sigma_{h}\right)$ of the gaussian $\left\{a_{n}\right\}$ type.

Proof. Condition (ii) is just the main part of the proof of Lemma 5 of [B-S (1981)]. To show that (iii) is true we should follow its steps more carefully. The main steps are certain approximations.

I) The calculation of $\psi_{n}^{(2)}(t)$ is restricted to sequences $\omega_{-n_{1}}, \ldots, \omega_{n+n_{1}}$, where $r_{ \pm}\left(\omega_{i}\right) \leqq 2 n_{1}+1=n_{2},\left(-n_{1} \leqq i \leqq n+n_{1}\right)$. By Property TB, the probability of these sequences is not less than $1-\left(n+n_{1}\right) \lambda_{1}^{n_{2}}$ and the new integral is

$$
\psi^{(3)}(t)=\sum_{r_{ \pm}\left(\omega_{i}\right) \leqq n_{2}} \exp \left[i\left(\sigma_{h} n\right)^{-1 / 2} \sum_{s=1}^{p} \sum_{k \in \Delta(1)} h_{n_{1}}\left(T_{0}^{k} \omega\right)\right] \mu_{0}\left(\omega_{-n_{1}}, \ldots, \omega_{n+n_{1}}\right) .
$$

It is then an easy exercise to show that

$$
\left|\psi^{(2)}(t)-\psi^{(3)}(t)\right| \leqq \operatorname{const}\left(n+n_{2}\right) \lambda_{1}^{n_{2}}
$$

II) Now $\mu_{0}$ is substituted by a Markov measure $\mu_{0}^{(1)}$ of memory $n_{2}$ while the characteristic function $\psi_{n}^{(3)}(t)$ goes over to $\psi_{n}^{(4)}(t)$. From Property MA, [B-S (1981)] obtains the bound

$$
\begin{aligned}
\left|\psi_{n}^{(3)}(t)-\psi_{n}^{(4)}(t)\right| & \leqq \operatorname{Var}\left(\mu_{0}, \mu_{0}^{(1)}\right) \\
& \leqq \varepsilon_{n}=n \log \left(1+\lambda_{4}^{n_{1}}\right)-2 n \log \left(1-\mu_{0}\left(\bar{V}_{n_{1}}\right)\right) .
\end{aligned}
$$

III) Finally, by using Property D, one approximates $\psi^{(4)}(t)$ by the characteristic function $\psi_{n}^{(5)}(t)$ of a product of $p$ independent, identically distributed random variables, namely

$$
\psi_{n}^{(5)}(t)=\prod_{r=1}^{p} E^{(1)} \exp \left[i\left(\sigma_{h} n\right)^{-1 / 2} t \sum_{k \in \Delta_{r}^{(1)}} h_{n_{1}}\left(T_{0}^{k} \omega\right)\right],
$$


where the expectation is taken with respect to $\mu_{0}^{(1)}$. According to [B-S (1981)]

$$
\left|\psi_{n}^{(4)}(t)-\psi_{n}^{(5)}(t)\right| \leqq p \cdot\left[\lambda_{3}^{\prime B_{n} / n_{1}}+n^{2} \mu_{0}\left(\bar{V}_{n_{1}}\right)\right]
$$

with some $\lambda_{3}^{\prime} \in(0,1)$.

We first claim that there exist positive constants $\varepsilon$ and $c$ such that for every $n$ and $|t|<\varepsilon n^{1 / 2}$,

$$
\left|\psi^{(5)}(t)\right| \leqq e^{-c t^{2}} .
$$

Indeed, [B-S (1981)] proves that

and

$$
\begin{gathered}
\left|E^{(1)} h_{n_{1}}\left(\omega_{-n_{1}}, \ldots, \omega_{n_{1}}\right)\right| \leqq \text { const } n^{2} \lambda_{20}^{n^{\lambda_{21}}}, \\
E^{(1)}\left(\sum_{k \in \Delta_{r}^{(1)}} h_{n_{1}}\left(T_{0}^{k} \omega\right)\right)^{2} \sim \sigma_{h} A_{n},
\end{gathered}
$$

$$
E^{(1)}\left(\sum_{k \in \Delta} h_{\frac{(1)}{r}} h_{n_{1}}\left(T_{0}^{k} \omega\right)\right)^{4} \leqq \text { const } A_{n}^{2} .
$$

Consequently, by using the Taylor expansion and Hölder inequality one obtains (3.5) easily.

In order to obtain possibly sharper results our choice for $A_{n}, B_{n}$, and $n_{1}$ differs from the standard one. Put $A_{n}=n \log ^{-\alpha} n, B_{n}=\log ^{\beta} n, n_{1}=\log ^{\gamma} n$ with $0<\gamma<\beta$. Then $p=\log ^{\alpha} n$ and by (3.3) and (3.4),

and

$$
\begin{aligned}
& \left|\psi_{n}^{(4)}(t)-\psi_{n}^{(5)}(t)\right| \leqq \text { const } \log ^{\alpha} n\left[\left(\lambda_{3}^{\prime}\right)^{\log ^{\beta-\gamma_{n}}}+n^{2} 2 \log ^{\gamma} n \lambda_{20}^{\log \gamma \lambda_{21 n}}\right], \\
& \left|\psi_{n}^{(3)}(t)-\psi_{n}^{(4)}(t)\right| \leqq \text { const } n\left[\lambda_{4}^{\log \gamma_{n}}+\log ^{\gamma} n \lambda_{20}^{\log \gamma^{\lambda_{21} n}}\right],
\end{aligned}
$$

$$
\left|\psi^{(2)}(t)-\psi^{(3)}(t)\right| \leqq \text { const } n \lambda_{1}^{2 \log ^{\gamma} n} .
$$

Reminding ourselves that $\lambda_{21} \in(0,1)$ can be chosen arbitrarily, we can see that all these differences tend to zero faster than any power of $n$ only if $\gamma>1$ and $\beta-\gamma>1$.

Now from (3.5) and (3.7) we obtain by a simple calculation that there exist constants $A$ and $D$ such that for

$$
A \leqq|t| \leqq \varepsilon n^{1 / 2}, \quad \psi_{n}^{(2)}(t) \leqq \frac{D}{t^{2}} .
$$

Hence the lemma.

Return to the proof of Theorem 3.1. Fix $\delta>0$. Denote

$$
\eta_{n}=\sum_{s=1}^{p}\left[\sum_{k \in \Delta_{s}^{(2)}} h\left(T_{0}^{k} \omega\right)+\sum_{k \in \Delta\left(\frac{1}{s}\right)}\left(h\left(T_{0}^{k} \omega\right)-h_{n_{1}}\left(T_{0}^{k} \omega\right)\right)\right],
$$

and observe that $\psi_{n}(t)$ is the characteristic function of $S_{n}+\eta_{n}$. By (3.2) the second sum in (3.8) is bounded by a sequence tending to 0 since $\gamma>1$. Concentrate on the first sum. If we used (3.1) and our forthcoming argument based on Lemma 3.4, then we could immediately obtain the QLCLT of the gaussian $\left\{\log ^{2+\delta} n\right\}$ type. To obtain a better result observe that the first sum is the sum of $p$ almost independent random variables. Indeed, the word by word repetition of our approximation used to treat $S_{n}$ gives us that we can define a new measure such that (i) its distance in variation from $\mu_{0}$ on the words $\omega_{-n_{1}}, \ldots, \omega_{n+n_{1}}$ tends to zero faster than any power of $n$, and (ii) the bounded summands $\vartheta_{k}=\sum_{\left.k \in \Delta \varsigma_{s}^{2}\right)} h\left(I_{0}^{k} \omega\right), 1 \leqq s \leqq p$ become independ- 
ent and identically distributed. Thus, with respect to the new measure the summands satisfy the conditions of Bernstein's exponential inequality [I-L (1971)] and

$$
P\left(\left|\sum_{k=1}^{p} \vartheta_{k}\right|>\log ^{1+\delta / 2} n\right) \leqq \exp \left(-\frac{1}{2 \sigma_{h}} \log ^{2+\delta-\alpha-\beta} n\right),
$$

since by (3.6), $E^{(1)} \vartheta_{k}^{2} \sim \sigma_{h} B_{n}$. We can choose $\alpha=\frac{\delta}{3}, \beta=1+\frac{\delta}{3}$, and we obtain again that the exponential bound tends to zero faster than any power of $n$. Consequently we can conclude that except for a negligible set $\left|\eta_{n}\right| \leqq \log ^{1+\delta / 2} n$. In fact, sets of probabilities tending to zero faster than any power of $n$ can be neglected in our considerations, since we are interested in sets where probabilities are larger than $n^{-d / 2}$.

Finally we introduce independent pairs of gaussian random variables $\zeta_{n}$ and $\tilde{\zeta}_{n}$ with variance $a_{n}^{2}=\log n$, say, and $b_{n}^{2}=\log ^{2+2 \delta} n-a_{n}^{2}$ respectively, and we also assume that the pairs $\left(\zeta_{n}, \widetilde{\zeta}_{n}\right)$ and $\left(S_{n}, \eta_{n}\right)$ are independent.

By Lemma (3.3) $T_{n}=S_{n}+\zeta_{n}$ obeys the $\operatorname{LCLT}\left(\sigma_{h}\right)$. The statement of Theorem 3.1 is a straightforward consequence of the following lemma.

\section{Lemma 3.4. Suppose that}

(i) the random variables $T_{1}, T_{2}, \ldots$ satisfy the $\operatorname{LCLT}(\sigma), \sigma>0$;

(ii) $\eta_{n}$ is a sequence of random variables with $P\left(\left|\eta_{n}\right| \leqq d_{n}\right)=1$;

(iii) $\bar{\zeta}_{n}$ is a sequence of gaussian random variables, independent of $T_{n}$ and $\eta_{n}$ such that $E \widetilde{\zeta}_{n}=0, E \widetilde{\zeta}^{2}=\widetilde{b}_{n}^{2}$;

(iv) $\tilde{d}_{n}=o\left(\tilde{b}_{n}\right), \tilde{b}_{n}=o\left(n^{1 / 2}\right)$.

Then $Z_{n}=T_{n}+\eta_{n}+\widetilde{\xi}_{n}$ satisfies the $\operatorname{LCLT}(\sigma)$, too.

Proof. Denote the distrihution of $n^{-1 / 2} T_{n}$ by $F_{n}$, and let $b_{n}=n^{-1 / 2} \tilde{b}_{n}, d_{n}=n^{-1 / 2} \tilde{d}_{n}$. Decompose the real axis into intervals $J_{\ell}=\left[\ell d_{n},(\ell+1) d_{n}\right)$, of length $-\infty<\ell<\infty$. With no loss of generality we shall prove that for the probability density $p_{n}(x)$ of $n^{-1 / 2} Z_{n}$

$$
p_{n}(0) \rightarrow \varphi_{\sigma}(0) \text {. }
$$

A simple thought shows that

$$
\begin{aligned}
\sum_{\ell=0}^{\infty} F_{n}\left(J_{\ell}\right) \varphi_{b_{n}^{2}}\left((\ell+2) d_{n}\right) & \\
& +\sum_{\ell=-1}^{-\infty} F_{n}\left(J_{\ell}\right) \varphi_{b_{n}^{2}}\left((\ell-1) d_{n}\right) \leqq p_{n}(0) \\
\leqq & \left(F_{n}\left(J_{-1}\right)+F_{n}\left(J_{0}\right)\right) \varphi_{b_{n}^{2}}(0)+\sum_{\ell=1}^{\infty} F_{n}\left(J_{\ell}\right) \varphi_{b_{n}^{2}}\left((\ell-1) d_{n}\right) \\
& +\sum_{\ell=-2}^{-\infty} F_{n}\left(J_{\ell}\right) \varphi_{b_{n}^{2}}\left((\ell+2) d_{n}\right) .
\end{aligned}
$$

We claim that the difference of the two bounds given for $p_{n}(0)$ tends to zero. In fact, this statement follows from

$$
\lim _{n \rightarrow \infty} \int_{0}^{\infty}\left(\varphi_{b_{n}^{2}}(y)-\varphi_{b_{n}^{2}}\left(y+d_{n}\right)\right) d F_{n}(y)=0 .
$$


Fix an $\omega>0$. This integral equals to

$$
\begin{aligned}
& (2 \pi)^{-1 / 2}\left(\int_{0}^{\omega b_{n}^{2} d_{n}^{-1}} b_{n}^{-1} e^{-\left(2 b_{n}^{2}\right)^{-1} y^{2}}\left\{1-\exp \left[-b_{n}^{-2} y d_{n}-\left(2 b_{n}^{2}\right)^{-1} d_{n}^{2}\right]\right\} d F_{n}(y)\right. \\
& \left.+\int_{\omega b_{n}^{2} d_{n}^{-1}}^{\infty} b_{n}^{-1} e^{-\left(2 b_{n}^{2}\right)^{-1} y^{2}}\left\{1-\exp \left[-b_{n}^{-2} y d_{n}-\left(2 b_{n}^{2}\right)^{-1} d_{n}^{2}\right]\right\} d F_{n}(y)\right)=I_{1}+I_{2} .
\end{aligned}
$$

In the domain $y>\omega b_{n}^{2} d_{n}^{-1}$ the gaussian density

$$
(2 \pi)^{-1} b_{n}^{-1} \exp \left[\left(-2 b_{n}^{2}\right)^{-1} y^{2}\right]
$$

becomes exponentially small and $I_{1} \rightarrow 0$. To bound $I_{2}$ we first use the fact that the densities $\frac{d F_{n}(y)}{d y}$ are uniformly bounded since $n^{-1 / 2} T_{n}$ obeys the $\operatorname{LCLT}(\sigma)$. Consequently, by taking the integral with respect to $d y$ we obtain an upper bound. Then, by the substitution $u=b_{n}^{-1} y$, this bound becomes

$$
\Phi_{1}\left(\omega b_{n}^{-1} d_{n}\right)-\Phi_{1}\left(\omega b_{n}^{-1} d_{n}-b_{n}^{-1} d_{n}\right)+\Phi_{1}(0)-\Phi_{1}\left(-b_{n}^{-1} d_{n}\right),
$$

which tends to zero in view of (iv).

\section{Quasi-Recurrence of the Planar Lorentz Process}

The techniques developed in the preceding section enable us to prove a weaker result than Pólya's classical recurrence theorem, only. As we shall see in the course of the proof, the principal obstacle in proving the exact recurrence is caused by the "lacunarity" in property $D$ of the Markov partition: as we have no inference about probabilities that $\sum_{k=0}^{n-1} \mathbf{h}\left(T_{0}^{k} \omega\right)$ lies in regions of size $\log ^{k} n, \kappa<1$. Therefore we introduce the notion of quasi-recurrence. Suppose $\left\{a_{n}\right\}$ is an increasing sequence of positive numbers.

Definition 4.1. We say that a sequence $S_{n}$ of random vectors is quasi-recurrent of the $\left\{a_{n}\right\}$ type if

$$
P\left\{S_{n} \in Q\left(a_{n}\right) \text { i.o. }\right\}=1 \text {. }
$$

Theorem 4.2. The two-dimensional Lorentz process is quasi-recurrent of the $\left\{\log ^{e} n\right\}$ type for every $\varrho>\frac{3}{2}$.

Proof. The idea of the proof consists of two ingredients formulated as two lemmas.

Lemma 4.3. The sequence of events

$$
A_{n}=\left\{\sum_{k=0}^{n-1} \mathbf{h}\left(T_{0}^{k} \omega\right) \in Q\left(\log ^{\varrho} n\right)\right\}
$$


fulfills the conditions of Lamperti's theorem [S (1964), p. 317],

$$
\begin{gathered}
\sum_{k=1}^{\infty} \mu_{0}\left(A_{k}\right)=\infty \\
\liminf _{n \rightarrow \infty} \frac{\sum_{j, k=1}^{n} \mu_{0}\left(A_{j} A_{k}\right)}{\left(\sum_{k=1}^{n} \mu_{0}\left(A_{k}\right)\right)^{2}}<c,
\end{gathered}
$$

consequently

$$
\mu_{0}\left\{A_{k} \text { i.o. }\right\}>\frac{1}{c}
$$

Lemma 4.4. The event

$$
\hat{\mathfrak{A}}_{v}=\left\{\forall \varepsilon, 0<\varepsilon<\frac{v}{4}, \sum_{k=0}^{n-1} \mathbf{h}\left(T_{0}^{k} \omega\right) \in Q\left((v+\varepsilon) \log ^{\varrho} n\right) \text { i.o. }\right\}
$$

is $T_{0}$ invariant, therefore by the ergodicity of $T_{0}, \mu_{0}\left(\hat{\mathfrak{A}}_{v}\right)= \begin{cases}0 & \text { or } \\ 1 & \end{cases}$

Proof of Lemma 4.3. First observe that condition (4.1) is an obvious consequence of the following rough estimate, derivable from Corollary 3.2: there exist two positive constants $C_{1}$ and $C_{2}$ depending on the function $\mathbf{h}$ only such that

$$
\frac{C_{1} \log ^{2 \varrho}}{n} \leqq \mu_{0}\left\{\sum_{k=0}^{n-1} \mathbf{h}\left(T_{0}^{k} \omega\right) \in Q\left(\log ^{\varrho} n\right)\right\} \leqq \frac{C_{2} \log ^{2 \varrho} n}{n}
$$

To prove (4.2) we have to repeat the Markov approximation of $\sum_{k=0}^{n-1} \mathbf{h}\left(T_{0}^{k} \omega\right)$ to obtain i.i.d. random variables as was done in the proof of Theorem 3.1 and to apply estimate (4.3).

If, in (4.2), $\mathbf{h}\left(T_{0}^{k} \omega\right)$ is substituted by $\mathbf{h}_{n_{1}}\left(T_{0}^{k} \omega\right), n_{1}=\log ^{\gamma} n, \varrho>\gamma>1$, then the orders of the probabilities $P\left(A_{k}\right), P\left(A_{j} A_{k}\right)$ do not change.

The same is true when the calculation of these probabilities is restricted to sequences $\omega_{-n_{1}}, \ldots, \omega_{n+n_{1}}$, where $r_{ \pm}\left(\omega_{i}\right) \leqq 2 n_{1}+1=n_{2}$, and the measure $\mu_{0}$ is substituted by a Markov measure $\mu_{0}^{(1)}$ of memory $n_{2}$.

Now we decompose the sum in the numerator of (4.2) as follows

$$
\begin{aligned}
\sum_{1 \leqq j, k \leqq n} \mu_{0}\left(A_{j} A_{k}\right)= & \sum_{1 \leqq k \leqq n} \mu_{0}\left(A_{k}\right)+2 \sum_{\substack{k-j<2 \log ^{\beta} n \\
1 \leqq j<k \leqq n}} \mu_{0}\left(A_{j} A_{k}\right) \\
& +2 \sum_{\substack{k-j \geqq 2 \log ^{\beta} n \\
1 \leqq j<k \leqq n}} \mu_{0}\left(A_{j} A_{k}\right) .
\end{aligned}
$$

The first and second terms can be bounded by

$$
\begin{aligned}
4 \log ^{\beta} n \sum_{1 \leqq k \leqq n} \mu_{0}\left(A_{k}\right) & <4 C_{2} \log ^{\beta} n \sum_{1 \leqq k \leqq n} \frac{\log ^{2 \varrho} k}{k} \\
& \sim \frac{4}{2 \varrho+1} C_{2} \log ^{2 \varrho+\beta+1} n .
\end{aligned}
$$


If $k-j>2 \log n$, then

$$
\begin{aligned}
\mu_{0}\left(A_{j} A_{k}\right)= & \mu_{0}\left(\left\{\sum_{i=0}^{j-1} \mathbf{h}\left(T_{0}^{i} \omega\right) \in Q\left(\log ^{e} j\right)\right\}\right. \\
& \left.\cap\left\{\sum_{i=0}^{k-1} \mathbf{h}\left(T_{0}^{i} \omega\right) \in Q\left(\log ^{e} k\right)\right\}\right)
\end{aligned}
$$

can be bounded from above by

$$
\begin{aligned}
& g_{j k} \stackrel{\text { def }}{=} 3^{2} \cdot \mu_{0}\left(\left\{\sum_{i=0}^{j-1} \mathbf{h}\left(T_{0}^{i} \omega\right) \in Q\left(\log ^{\varrho} n\right)\right\}\right. \\
&\left.\cap\left\{\sum_{i=j+\left[\log ^{\varrho} n\right]}^{k} \mathbf{h}\left(T_{0}^{i} \omega\right) \in Q\left(\log ^{\varrho} n\right)\right\}\right) .
\end{aligned}
$$

Property D of the Markov partition provides that the events

$$
\left\{\sum_{i=0}^{j-1} \mathbf{h}\left(T_{0}^{i} \omega\right)\right\} \text { and }\left\{\sum_{i=j+\left[\log ^{2} n\right]}^{k} \mathbf{h}\left(T_{0}^{i} \omega\right)\right\}
$$

are almost independent, i.e. the transition to an independent measure does not change the order of $g_{j k}$. So by (4.3),

$$
g_{j k} \leqq C_{2}^{2} \log ^{4 e} n \cdot \frac{1}{j} \cdot \frac{1}{k-j} .
$$

Using the identity $\frac{1}{j} \cdot \frac{1}{k-j}=\frac{1}{k}\left(\frac{1}{j}+\frac{1}{k-j}\right)$ it is easy to check that

$$
\sum_{1<j<k \leqq n} g_{j k} \leqq C_{2}^{2} \log ^{4 \varrho+2} n .
$$

The bound (4.3) gives that

$$
C_{1}^{2}\left(\sum_{k=1}^{n} \log ^{2 \varrho} k\right)^{2} \sim\left(\frac{C_{1}}{\varrho+1}\right)^{2} \log ^{4 \varrho+2} n
$$

is a lower bound for the denominator of (4.2). It follows from (4.4), (4.5), and (4.6) that the numerator and denominator of (4.2) have the same order when $n \rightarrow \infty$. Q.E.D.

The statement of Lemma 4.4 is a straightforward consequence of the definition of $\hat{\mathfrak{A}}_{v}$.

Obviously the proof of Lemma 4.3 can be carried out for every

$$
\mathfrak{A}_{v} \underset{ }{\text { def }}=\left\{\sum_{k=0}^{n-1} \mathbf{h}\left(T_{0}^{k} \omega\right) \in Q\left(v \log ^{\varrho} n\right) \text { i.o. }\right\},
$$

consequently for every $v, \mu_{0}\left(\mathfrak{U}_{v}\right)>0$. On the other hand

i.e.

$$
\mathfrak{A}_{1 / 4} \cong \hat{\mathfrak{A}}_{1 / 2} \cong \mathfrak{U}_{1},
$$

$$
0<\mu\left(\mathfrak{U}_{1 / 4}\right) \leqq 1 \leqq \mu\left(\mathfrak{U}_{1}\right) .
$$

This last inequality proves Theorem 4.2. 
Acknowledgements. The authors express their sincere gratitude to Ya. G. Sinai for directing their interest to the problem of recurrence and for his constant attention to their work.

Thanks are due to P. Révész for informing them about Lamperti's Borel-Cantelli lemma.

\section{References}

[B-S (1980)] Bunimovich, L.A., Sinai, Ya.G.: Markov partitions for dispersed billiards. Commun. Math. Phys. 78, 247-280 (1980)

[B-S (1981)] Bunimovich, L.A., Sinai, Ya.G.: Statistical properties of Lorentz gas with periodic configuration of scatterers. Commun. Math. Phys. 78, 479-497 (1981)

[C (1982)] Chernov, N.I.: Construction of transversal fibers for many-dimensional nonstrictly-dispersing billiards (in Russian). Funkcionalni Analiz. 16, 35-46 (1982)

[C-F-S (1982)] Cornfeld, I.P., Fomin, S.V., Sinai, Ya.G.: Ergodic theory. Berlin, Heidelberg, New York: Springer 1982

[I-L (1971)] Ibragimov, I.A., Linnik, Yu.V.: Independent and stationary sequences of random variables Groningen: Wolters-Noordhoff 1971

[K-Sz (1983)]a) Krámli, A., Szász, D.: Random walks with internal degrees of freedom. I. Local limit theorems. Z. Wahrscheinlichkeitstheorie 63, 85-95 (1983)

[K-Sz (1983)]b) Krámli, A., Szàsz, D.: Central limit theorem for the Lorentz process via perturbation theory. Commun. Math. Phys. 91, 519-528 (1983)

[S (1964)] Spitzer, F.: Principles of random walks. New York: Van Nostrand 1964

Communicated by Ya. G. Sinai

Received August 10, 1984 